\title{
Microglial/macrophage accumulation during cuprizone-induced demyelination in C57BL/6 mice
}

\author{
M.M. Hiremath ${ }^{a, b}$, Y. Saito ${ }^{\text {b,c,1 }}$, G.W. Knapp ${ }^{\text {b,2 }}$, J.P.-Y. Ting ${ }^{\text {a,d }}$, K. Suzuki ${ }^{\text {b,c }}$, \\ G.K. Matsushima ${ }^{\mathrm{a}, \mathrm{b}, \mathrm{e}, *}$ \\ a Department of Microbiology and Immunology, University of North Carolina at Chapel Hill, Chapel Hill, NC 27599-7290, USA \\ ${ }^{\mathrm{b}}$ UNC Neuroscience Center, University of North Carolina at Chapel Hill, Chapel Hill, NC 27599-7250, USA \\ ${ }^{\mathrm{c}}$ Department of Pathology and Laboratory Medicine, University of North Carolina at Chapel Hill, Chapel Hill, NC 27599-7525, USA \\ ${ }^{\mathrm{d}}$ UNC Lineberger Comprehensive Cancer Center, University of North Carolina at Chapel Hill, Chapel Hill, NC 27599-7595, USA \\ ${ }^{\mathrm{e}}$ Program for Molecular Biology and Biotechnology, University of North Carolina at Chapel Hill, Chapel Hill, NC 27599, USA
}

Received 25 February 1998; revised 4 June 1998; accepted 18 June 1998

\begin{abstract}
To study microglial/macrophage infiltration, a cuprizone-induced model for demyelination in C57BL/6 mice was established. Cuprizone is known to cause demyelination in Swiss mice, however, cuprizone-induced demyelination in C57BL/6 mice has not been previously described. Induction of demyelination in C57BL/6 mice enables examination of the function of microglia/macrophage through comparative analyses of syngeneic mice with various targeted genetic mutations. In this report, cuprizone-induced demyelination is easily inducible, localized, and predictable. Concurrent with the initiation of demyelination, we noted microglial/macrophage accumulation and changes in astrocyte morphology. Astrogliosis promptly followed microglia/macrophage recruitment. These observations suggested that microglia/macrophage actively contribute to the demyelination process. C 1998 Elsevier Science B.V. All rights reserved.
\end{abstract}

Keywords: Microglia/macrophage; Astrocytes; Immunology; Central nervous system (CNS); Brain

\section{Introduction}

The central nervous system (CNS) was thought to be an immune privileged site; however, the presence of MHC class II-expressing cells of bone marrow origin in murine brains would contradict this supposition (Ting et al., 1981; Hickey and Kimura, 1988). It is now known that microglia and infiltrating macrophages are present in a variety of CNS disorders (McGeer et al., 1988a; McGeer et al., 1988b; Mattiace et al., 1990; Styren et al., 1990; Ohno et al., 1992; Perlmutter et al., 1992; Tomimoto et al., 1993). In conditions such as multiple sclerosis (MS) and amyotrophic lateral sclerosis (ALS), a large presence of infil-

\footnotetext{
* Corresponding author. Tel.: +1919 9660408; fax: + 1919 9661844; e-mail: gkmats@med.unc.edu

${ }^{1}$ Present address: Department of Otolaryngology, Japanese Red Cross Society, Wakayama Medical Center, 4-20 Komatsubara-dori, Wakayama, 640, Japan.

${ }^{2}$ Present address: Genetic and Cellular Toxicity Branch, MD\#68, USEPA, Research Triangle, NC 27711, USA.
}

trating T-lymphocytes are found at sites of microglia or macrophage activation (Traugott et al., 1983; Zamvil and Steinman, 1990; Martin et al., 1992; Li et al., 1993; Tomimoto et al., 1993). In contrast, a number of non-immunologic neurological diseases exhibit microglia/macrophages without obvious infiltration of T-lymphocytes (McGeer et al., 1988a; Mattiace et al., 1990; Styren et al., 1990; Perlmutter et al., 1992). In addition, in certain areas of demyelinating lesions in brains from MS patients, foamy microglia exist in the absence of significant T-cell infiltration (Raine, 1994). We have previously implicated microglia/macrophages as effector cells that directly contribute to demyelination in twitcher mice, a murine model for human Krabbe's disease (Matsushima et al., 1994). Thus, in demyelinating diseases, microglia/macrophage may have functions that exacerbate the demyelination process (Matsushima et al., 1994), independent of activating T-lymphocytes.

Demyelination is evident in the brains of people afflicted with various neurological disorders (Ludwin, 1981). 
Experiments addressing demyelination have been performed by establishing and using various models, including: Wallerian degeneration (Bruck, 1997); CSF barbotage (Bunge et al., 1961); experimental allergic encephalitis (EAE) (Lampert, 1965, 1967; Prineas et al., 1969); cord compression (Gledhill et al., 1973); intracerebral or intraspinal injections of diphtheria toxin (Wisniewski and Raine, 1971; Harrison et al., 1972); cyanide intoxication (Hirano et al., 1968); and cuprizone, or ethidium bromide intoxication (Suzuki and Kikkawa, 1969; Blakemore, 1973; Yajima and Suzuki, 1979). Microglial/macrophage presence in all of these various models for demyelination suggests an important function for these cells.

Copper is an essential trace element for a number of metalloenzymes (Walshe, 1995) including copper-zinc superoxide dismutase (CuZnSOD1) and ceruloplasmin (Zlotkin et al., 1995). Dietary changes in copper with copper-chelators such as cuprizone produce copper deficiency and CNS demyelination (Suzuki and Kikkawa, 1969; Blakemore, 1972; Blakemore, 1973; Ludwin, 1978). Well documented consequences of copper deficiency induced by cuprizone are the degeneration of oligodendrocytes and formation of megamitochondria in the liver possibly resulting from metabolic alterations in cytochrome- $C$ levels (Suzuki, 1969; Suzuki and Kikkawa, 1969). Oligodendrocytes appear to be particularly sensitive to low doses of cuprizone with minimal damage to other cell types (Blakemore, 1973; Ludwin, 1978; Komoly et al., 1987; Fujita et al., 1990; Cammer and Zhang, 1993). This decreased level of energy presumably leads to oligodendrocytic dysfunction and eventually to demyelination. Demyelination is accompanied by the appearance of microglia and by astrogliosis (Blakemore, 1972; Blakemore, 1973; Ludwin, 1978); however, there is no obvious Tlymphocyte infiltration presumably due to an intact blood-brain barrier (Bakker and Ludwin, 1987; Kondo et al., 1987). Thus, cuprizone-induced copper deficiency provides a model for demyelination that enables us to investigate the function of microglia/macrophages present in the demyelinating lesions without the confounding presence of T-cell infiltrates.

Cuprizone treatment of animals has been used as a model for investigating the changes or reactions of neuronal and glial cells accompanying the damage of oligodendrocytes in Swiss, CD1, and ICI mice (Blakemore, 1974; Ludwin, 1978; Bakker and Ludwin, 1987). Demyelination by cuprizone treatment has not been described in C57BL/6 mice; however, we report here conditions in which demyelination and microglia/macrophage accumulation can be induced in C57BL/6 mice without liver involvement. Although many studies demonstrate the toxic effects of cuprizone on the brain (Blakemore, 1973; Ludwin, 1978), little is known about the appearance of microglia and its correlation with neuropathology. This study focuses on microglial/macrophage accumulation into lesions of demyelination during cuprizone treatment of
C57BL/6 mice and documents the chronological changes with respect to microglia/macrophages and astrocytes in the corpus callosum where demyelination was greatest. Most notably, microglial/macrophage appearance at or adjacent to the affected sites in the CNS upon the induction of demyelination is documented. The findings reported are important in providing a model to test the function of genes in various genetically altered knockout or transgenic mice and rapidly assess significance in pathology.

\section{Materials and methods}

\subsection{Mice and tissue preparation}

C57BL/6J male mice at 5 to 6 weeks old were either purchased from the Jackson Laboratory (Bar Harbor, ME, USA) or bred and maintained in our pathogen-free animal facility at UNC. Only 8 to 10 weeks old age-matched animals were used in an experiment. In the dosage study, the mice were provided a powder feed containing $0.1 \%$, $0.2 \%, 0.3 \%, 0.4 \%, 0.5 \%$ and $0.6 \%$ cuprizone (Sigma, St. Louis, MO) for 6 weeks. Cuprizone was physically mixed into ground Breeder Chow 2000 (Purina, Richmond, IN) by a weight to weight ratio. The mice were monitored for clinical symptoms and sacrificed at 6 weeks of treatment to determine neuropathology and to conduct immunohistochemistry. For the chronology study, groups of at least four mice were fed a diet containing $0.2 \%$ cuprizone and sacrificed after 1, 2, 3, 4, 5 or 6 weeks of feeding.

Mice were anesthetized with Metafane (ScheringPlough, Omaha, NE) and perfused through the heart with $0.1 \mathrm{M}$ phosphate buffer (PB) followed by either $4 \%$ paraformaldehyde for paraffin embedding or $2 \%$ periodatelysine-paraformaldehyde (PLP) solutions for immunohistochemical analyses. The brains were further post-fixed in situ overnight at $4^{\circ} \mathrm{C}$ in the same fixative and removed. The cerebrum was coronally sectioned to expose the corpus callosum. In each experiment, the brains of two cuprizone-treated mice at each time point were embedded in paraffin for histological and immunocytochemical studies. Additional two brains were used for frozen section immunocytochemistry as described below. The paraffin embedded tissues were sectioned at $5 \mu \mathrm{m}$ in thickness at the level of section 251 according to mouse atlas by Sidman et al. (1971). For routine histology, the sections were stained with hematoxylin and eosin (H\&E) and Luxol fast blueperiodic acid Schiff (LFB-PAS) stains. In addition, liver tissue was paraffin embedded and stained with $\mathrm{H} \& \mathrm{E}$ to determine toxicity to hepatocytes in cuprizone dosage studies. Morphology of the hepatocytes was observed at 400$600 \times$ and structures consistent with megamitochondria were observed at $1000 \times$ under oil immersion.

In order to evaluate demyelination, three independent readers in a double-blind fashion scored LFB-PAS-stained 
sections from zero to three. Zero was equivalent to the myelin status of a mouse not treated with cuprizone, whereas a score of three was total demyelination usually observed at 6 weeks of treatment with cuprizone. A score of one is equivalent to demyelination of one-third of the fibers of the myelin tract, while a score of two is equivalent to demyelination of two thirds of the fibers of the myelin tract. Intermediate scores for demyelination are representative of intermediate percentages of demyelinated fibers in the myelin tract. In addition, cellularity was scored in these samples as an estimate of the number of cells infiltrating areas of demyelination. The estimate of the number of cells infiltrating demyelinating regions was based on the scoring of nuclei on a scale of zero to three, where zero represented few nuclei as in the untreated animal and three represented the heavy accumulation of nuclei in the myelin tract following complete demyelination. To verify the degree of demyelination and glial cell response, paraffin sections of the cerebrum were processed for immunocytochemical detection of the myelin basic protein (MBP) with the SMI 99 antibody (1:1000 dilution) (Sternberger Monoclonals, Bethesda, MD), of astrocytes with antibody to glial fibrillary acidic protein (GFAP; 1:1000 dilution) (DAKO, Santa Barbara, CA), and of microglia/macrophages with a lectin RCA-1 (Sigma), using the Autoprobe III kit with streptavidin-conjugated peroxidase base (Biomed, Foster City, CA). All scores and quantitation of cells in each coronal section of the corpus callosum were typically conducted at two sites, immediately lateral of midline.

Frozen sections were cryoprotected in $0.1 \mathrm{M}$ PB containing $20 \%$ sucrose for 2 to 3 days. Serial $30-\mu \mathrm{m}$ coronal sections were cut with a cryostat. The sections were washed with several changes in phosphate-buffered saline (PBS), $\mathrm{pH} 7.4$, followed by $0.3 \%$ hydrogen peroxide to block endogenous peroxidase activity. After rinsing with PBS three times ( $3 \mathrm{~min}$ each), the sections were incubated in PBS with $10 \%$ normal rabbit serum for $20 \mathrm{~min}$ followed by primary anti-Mac-1 antibody (Boehringer Mannheim, Indianapolis, IN) treatment at a 1:500 dilution with PBS containing $0.2 \%$ Triton $\mathrm{X}$ (PBST) overnight at $4{ }^{\circ} \mathrm{C}$. The sections were then washed and sequentially incubated with a 1:200 dilution of biotinylated rabbit anti-rat IgG (Vector Laboratory, Burlingame, CA) in PBST for $2 \mathrm{~h}$. The sections were washed with PBS and the signal was amplified with the ABC-Elite reagent (Vector Laboratory, Burlingame, CA) for $1 \mathrm{~h}$ at the room temperature. The immunoproduct was then visualized with the DAB kit (Vector). The sections were dehydrated and mounted with permount (Fisher Scientific, Pittsburgh, PA).

\subsection{Microscopic morphometry}

The H\&E-stained sections were used for the quantitative analysis of cells infiltrating the corpus callosum during demyelination. Individual cells were counted based on nuclei (Smart and Leblond, 1961). All cells in the corpus callosum at the level of section 251 (Sidman et al., 1971) were counted manually using a morphometry system based on a Nikon FXA microscope (Garden City, NY) equipped with an Optronics TEC-470 CCD Video Camera System (Goleta, CA). Densitometric analysis of stained sections was also performed, and it confirmed the trends seen in the manual cell counts (data not shown). However, we have chosen to present our data on the basis of cell counts to emphasize the increased cellularity that accompanies cuprizone-induced demyelination. Images were captured on an Apple Macintosh 840 AV computer (Cupertino, CA) using a Scion LG-3 card (Fredrick, MD). Image processing, analyses, and measurements were carried out using the public domain NIH image program (http://rsb.info. nih.gov/nih-image/). The counted cells from treated animals were compared to control untreated animals and subjected to statistical analysis using the Student's $t$-test. The area of one visual field was $0.04669 \mathrm{~mm}^{2}$ on the video screen by $40 \times$ objectives. Cells in the median of the corpus callosum were counted. Immunostained $\mathrm{GFAP}^{+}$ astrocytes, Mac- $1^{+}$microglia/macrophages, and RCA-1 ${ }^{+}$ microglia/macrophages were also individually counted in the corpus callosum for each experimental group. Only cells that contained a nucleus, as indicated by hematoxylin counterstain, were counted. A minimum of two sections per animal were analyzed for scoring.

\subsection{Statistical analyses}

Data were plotted to verify that individual values within a group showed a symmetric U-shaped distribution indicative of normal distribution. Statistical comparisons were conducted with two-tailed Student's $t$-tests with unequal

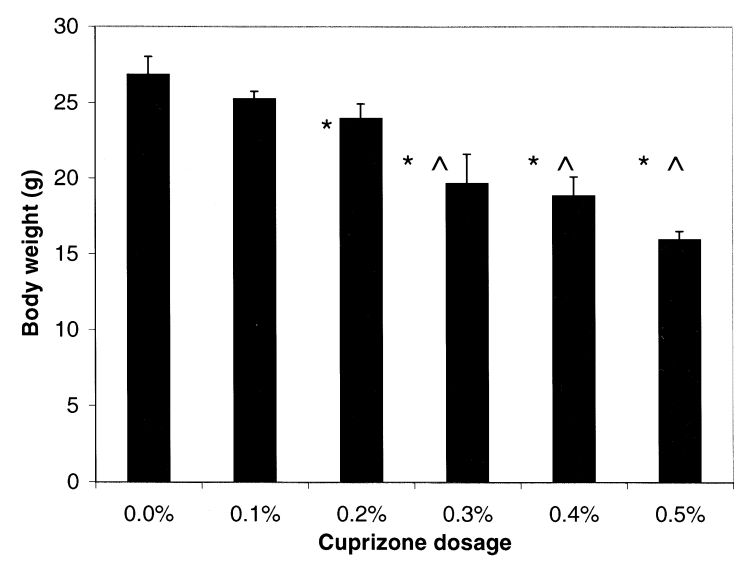

Fig. 1. Weight loss in cuprizone-treated C57BL/6 mice. Significant body weight loss compared to untreated mice was noted in mice treated with $0.2 \%$ or more of cuprizone for 6 weeks $(* p<0.05)$. Body weight loss from $0.2 \%$ compared to $0.3 \%$ or greater doses was also significant ( $p<0.05$, Student's $t$-test). Significance was based on a two-tailed Student's $t$-test assuming unequal variance. Histograms indicate the mean of four animals per group and error bars represent standard deviation. 
variance. Mean differences were considered significant if $p<0.05$ was computed. Treated mice were compared to either untreated mice or other sets of mice as indicated in the figure legends. The graphs represent mean values, and the error bars are indicative of the standard deviations.

\section{Results}

\subsection{Effect of cuprizone on the body weight and liver of C57BL / 6 mice}

Preliminary observation in the C57BL/6 mice showed mortality after 2 to 3 weeks at the $0.6 \%$ cuprizone dosage,
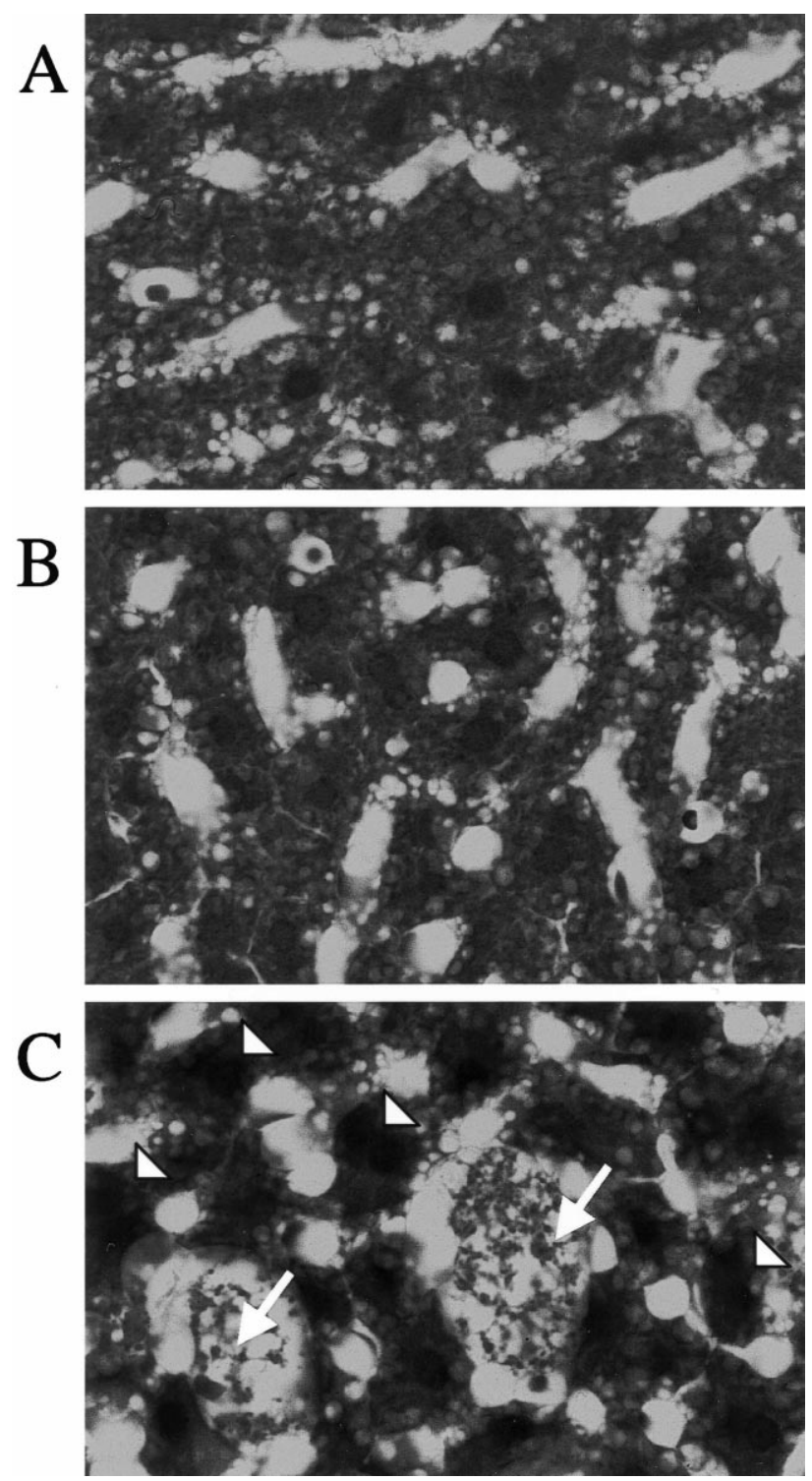

Fig. 2. Liver toxicity in cuprizone-treated C57BL/6 mice. Hematoxylin and eosin (H\&E)-stained, $5 \mu \mathrm{m}$ liver sections of untreated mice (A) were compared to liver sections of mice treated for 6 weeks with $0.2 \%$ cuprizone (B). Liver sections of mice treated with $0.4 \%$ or higher dose of cuprizone had indications of liver toxicity based on increased numbers of multinucleated hepatocytes (arrowheads), and degenerating cells (arrows) (C). Photomicrographs are shown at $600 \times$ magnification.

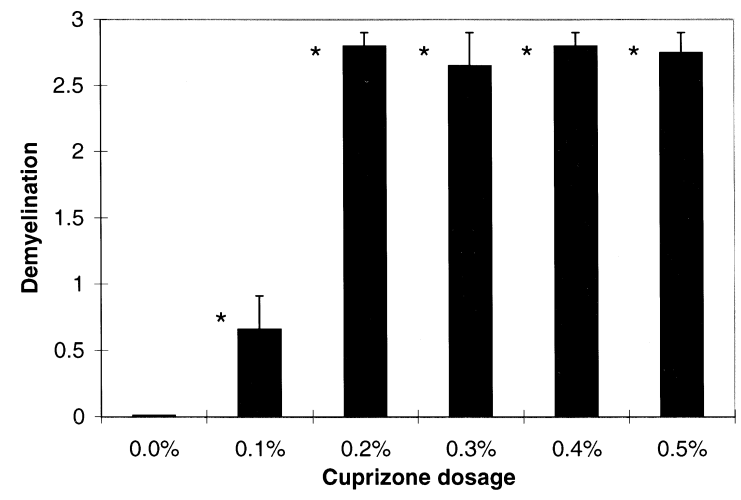

Fig. 3. Cuprizone dosage effects on demyelination in the C57BL $/ 6$ mouse. Upon 6 weeks of treatment with varying doses of cuprizone, $0.2 \%$ and greater doses of cuprizone resulted in almost complete demyelination. Although the $0.1 \%$ dose did not result in complete demyelination, the amount of demyelination was still significant when compared to the untreated mice $(* p<0.05)$. In these experiments, animals were perfused, their brains were removed, embedded in paraffin, cut at $5 \mu \mathrm{m}$, and stained with LFB-PAS. Coronal sections were scored, as mentioned in Section 2, for demyelination in the medial portion of the corpus callosum. Each group in this graph represents an average of four animals, and the error bars are the standard deviation for each group.

which is the dose used by other investigators to study different strains of mice (Blakemore, 1974; Ludwin, 1978; Komoly et al., 1987). Since $0.6 \%$ cuprizone was extremely toxic to the C57BL/6 mice, our subsequent studies did not use this high dose of cuprizone. Eight- to 10-week old age-matched mice were fed varying doses of cuprizone for 6 weeks and their body weight was taken following cuprizone treatment. The mean body weight of the mice fed with $0.1 \%$ cuprizone showed normal body weight gain and they behaved normally throughout the experimental period. However, the body weight of mice fed with $0.2 \%$ cuprizone for 6 weeks was significantly less than that of control untreated mice ( $p<0.05$, Student's $t$-test; Fig. 1). Mice fed $0.3 \%, 0.4 \%$, and $0.5 \%$ had extensive weight loss accompanied by a high degree of lethargy. Additionally, the mean body weight of mice fed $0.3 \%, 0.4 \%$, and $0.5 \%$ cuprizone was significantly less than mean body weight of mice on the $0.2 \%$ cuprizone diet $(p<0.05$, Student's $t$-test).

The significant differences in body weight at varying doses of cuprizone prompted an examination of liver toxicity in mice. Mice treated at varying doses from untreated to $0.5 \%$ were sacrificed at week six. Histological sections of liver were stained with $\mathrm{H} \& \mathrm{E}$ and analyzed for the presence of cellular degeneration, excessive numbers of multinucleated hepatocytes, and megamitochondria in hepatocytes. No overt abnormalities were observed in hepatocytes of animals treated with the $0.1 \%, 0.2 \%$, or $0.3 \%$ concentrations of cuprizone (Fig. 2A and B). Liver sections of C57BL/ 6 mice treated with $0.4 \%$ cuprizone for 6 weeks contained scattered hepatocytes with palcytoplasm containing fragmented organelles suggestive of degenera- 
tion and many multinucleated hepatocytes (Fig. 2C). Hepatocytes of $\mathrm{C} 57 \mathrm{BL} / 6$ mice treated with $0.5 \%$ cuprizone contained deeply eosinophilic enlarged globular structures consistent with megamitochondria (Suzuki, 1969) and increased numbers of multinucleated hepatocytes (data not shown). Thus, based on the body weight analysis data and the liver toxicity data, we have shown that the $0.2 \%$ cuprizone dose has an effect on the C57BL/6 mice. However, the wasting is minimal compared to greater doses of cuprizone, and there is no liver involvement.

\subsection{Dosage of cuprizone and time course of demyelination in C57BL/6 mice}

Cuprizone most notably induces demyelination in the cerebral white matter, particularly the corpus callosum and superior cerebellar peduncles (Blakemore, 1973; Ludwin,
1978). Demyelination was noted in the superior cerebellar peduncles; however, in this study, we have focused on demyelination in the corpus callosum where the extent of demyelination can be scored most easily and consistently. The degree of demyelination in the experimental animals was evaluated in the corpus callosum on LFB-PAS-stained and SMI 99(MBP) immunostained slides. In the dosage study, demyelination was incomplete by week six at the $0.1 \%$ dosage; however, demyelination approaching completion was evident 6 weeks after continuous administration of cuprizone at a dose of $0.2 \%$ or greater (Fig. 3). As shown in Fig. 4A, LFB-PAS-stained brain sections from untreated control mice show myelinated (blue) corpus callosum in contrast to animals treated for 6 weeks with $0.2 \%$ cuprizone that show complete demyelination (pink) in Fig. 4B. Additional SMI 99(MBP)-stained sections verified the severity of demyelination observed in LFB-PAS-

Fig. 4. Dose effects of cuprizone on demyelination of the corpus callosum. (A) LFB-PAS-stained section from untreated control C57BL/6 mice displaying (blue tract) myelinated corpus callosum. This section from an untreated mouse was scored as zero for demyelination. (B) LFB-PAS-stained section of the corpus callosum from a 6-week, $0.2 \%$ cuprizone-treated mouse indicating complete demyelination (pink tract as opposed to blue tract). This section was assigned a score of three for complete demyelination. Demyelination as ascertained by LFB-PAS was confirmed with antibody staining (SMI 99) for MBP. (C) SMI 99(MBP)-stained coronal section of an untreated C57BL/6 mouse exhibits intact, continuously stained fibers. (D) SMI 99(MBP)-stained coronal section of a C57BL/6 mouse treated with $0.2 \%$ cuprizone for 6 weeks shows rare and extremely scattered staining for MBP which is indicative of the absence of myelin. Arrows point to myelin fragments in the corpus callosum. All photomicrographs are shown at a $600 \times$ magnification. 


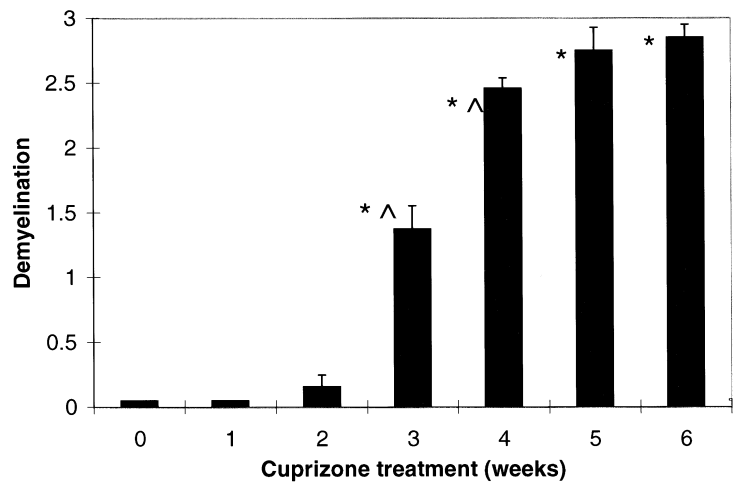

Fig. 5. Time course of demyelination in the corpus callosum of C57BL $/ 6$ mice. Histogram represents scores of coronal sections of the corpus callosum stained with LFB-PAS. Results are represented as averages of scores obtained in a double-blind manner by three independent investigators. Animals were treated with $0.2 \%$ cuprizone and sacrificed at various weekly time points. Significant demyelination compared to untreated mice was observed at week three through six $(* p<0.05)$. Additionally, a significant increase in demyelination compared to the previous week of treatment was noted at weeks three and four $(p<0.05)$. Each group is representative of an average demyelination score from four mice, and the error bars represent the standard deviation for each group.

stained brain sections. Brain sections of untreated control mice stained with SMI 99 showed consistent continuously staining fibers indicative of intact myelin fibers (Fig. 4C). Alternatively, brain sections of mice treated with $0.2 \%$ cuprizone for 6 weeks stained with the SMI 99 antibody showed very rare and scattered staining indicative of an absence of myelin (Fig. 4D). Mice treated with $0.3 \%$ cuprizone showed similar results. Although treatment of mice with $0.4 \%, 0.5 \%$, and $0.6 \%$ cuprizone resulted in demyelination of the corpus callosum, adverse liver toxicity was concomitantly observed (Fig. 2). Thus, it appeared that the $0.2 \%$ to $0.3 \%$ dose of cuprizone was the maximum dose tolerated by C57BL/ 6 mice which caused demyelination without liver toxicity. However, because the C57BL/6 mice treated with $0.3 \%$ cuprizone were lethargic and experienced a significant weight loss compared to mice on the $0.2 \%$ cuprizone diet (Fig. 1), subsequent studies on demyelination will be conducted at the $0.2 \%$ dose.

In the $0.2 \%$ cuprizone time course study, mice were analyzed at weekly intervals. No noticeable reduction in neither LFB-PAS nor SMI 99-staining was observed at the light microscopy level at week two of cuprizone treatment (Fig. 5). Demyelination of the corpus callosum was first detected at 3 weeks of treatment. Significant demyelination progressed rapidly over the following week of cuprizone feeding and by the 5th week of treatment demyelination approached 100\% (a score of three) within the corpus callosum. Thus, it appeared that $0.2 \%$ cuprizone treatment of the C57BL/6 mouse resulted in notable demyelination of the corpus callosum at approximately week three $(p<0.05)$ and progressed to nearly $100 \%$ by the 5th week.

\subsection{Increase in the number of cells within the corpus callosum during cuprizone treatment}

A noticeable increase in the number of cells was found in the corpus callosum of animals treated with increasing doses of cuprizone. The cellular response to cuprizone or to demyelination was analyzed by morphometric quantification of $\mathrm{H} \& \mathrm{E}$ sections from the corpus callosum. The $5 \mu \mathrm{m}$ paraffin sections were counted for total number of nuclei-containing cells that included microglia/macrophages, astrocytes, and oligodendrocytes. As shown in Fig. $6 \mathrm{~A}$, there was a significant increase in the number of cells in the corpus callosum in mice treated for 6 weeks with $0.1 \%$ to $0.5 \%$ cuprizone diet in comparison to untreated mice $(p<0.05)$. Peak cellularity was observed at the 6 week time point with the $0.4 \%$ dosage.
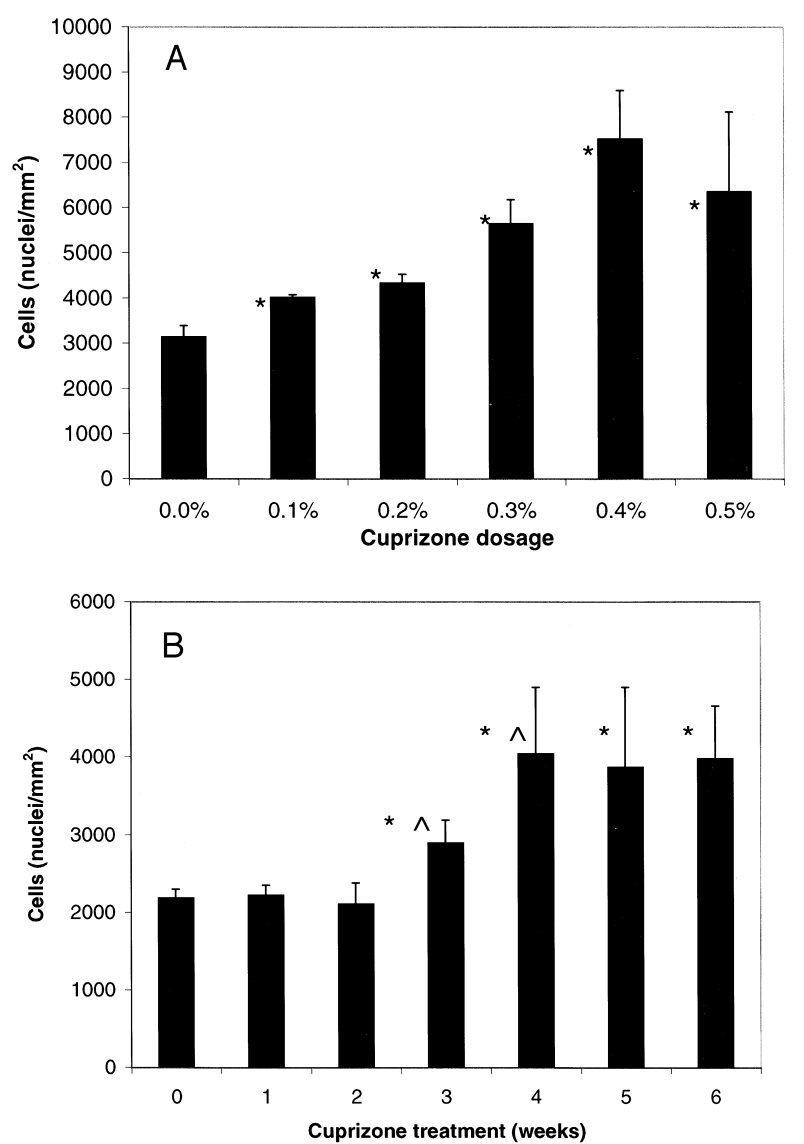

Fig. 6. Increase in cellularity during demyelination. Paraffin sections at 5 $\mu \mathrm{m}$ were stained with $\mathrm{H} \& \mathrm{E}$. Nuclei were counted as an indicator of cellularity in the corpus callosum. (A) A significant increase in cellularity was apparent at all doses of cuprizone treatment in C57BL/6 mice compared to brain sections of untreated mice $(* p<0.05)$. (B) Significant increase in cellularity was detected in the corpus callosum of cuprizone-treated mice compared to untreated mice at weeks three through six $(* p<0.05)$. Additionally, a significant increase in cellularity compared to the previous week of treatment was noted at weeks three and four $(p<0.05)$. Each group in this graph is an average of four animals. The error bars are representative of the standard deviation for each group. 
During the $0.2 \%$ cuprizone-treatment time course study, the number of cells present at the 1- and 2-week time points appeared to be identical to untreated control animals (Fig. 6B). A significant increase in the number of cells in the corpus callosum was first noted at the 3rd week of treatment $(p<0.05$; Fig. 6B). Cellularity significantly increased from the 3rd week of treatment and peaked at the 4 th week $(p<0.05)$. The peak level of cellularity was maintained at the 5th and 6th weeks of continuous cuprizone treatment.Thus, an increase in general cellularity in the corpus callosum correlated with apparent demyelination during cuprizone treatment.

\subsection{Astrogliosis and morphologic changes in astrocytes in the corpus callosum during cuprizone treatment}

An increase in astrogliosis has been previously reported during cuprizone intoxication (Elsworth and Howell, 1973). The status of astrocytes in the corpus callosum was assessed in C57BL/6 mice treated with cuprizone. At 6 weeks of treatment, brain sections stained for GFAP from C57BL/6 mice treated with varying doses of cuprizone treatment confirmed astrogliosis (Fig. 7A). The number of astrocytes increased significantly in all doses of cuprizone treatment when compared to untreated control animals. All doses appeared to induce similar numbers of astrocytes $(p>0.05)$.

In mice treated with $0.2 \%$ cuprizone, the number of astrocytes remained similar to basal levels during the 1st and 2nd week of cuprizone treatment (Fig. 7B). When significant levels of demyelination were observed at the 3rd-week time point, there was a corollary and significant increase in the number of astrocytes $(p<0.05)$, as shown by the increased numbers of nuclei staining positive for GFAP. In addition, dual staining with bromodeoxyuridine (BrdU) and GFAP in brain sections of cuprizone treated mice revealed that the astrocytes were proliferating (data not shown). GFAP ${ }^{+}$astrocytes continued to significantly $(p<0.05)$ increase in number in correlation to demyelination at the 4th week. The extent of astrogliosis was coincidental to extent of demyelination at the 5th and 6th weeks. Thus, part of the general increase in cellularity in Fig. 6 within the corpus callosum of C57BL/6-treated mice was contributed by GFAP-staining astroglia (Fig. 7A and B, Fig. 8), as previously demonstrated in other strains (Elsworth and Howell, 1973).

The appearance of the cellular processes of astrocytes was altered upon cuprizone treatment. In untreated control C57BL $/ 6$ mice, $\mathrm{GFAP}^{+}$astrocytic cells had delicate cellular processes (Fig. 8A). In contrast, astrocytes were hypertrophied with thick processes and were markedly increased in the brain of all experimental mice (Fig. 8B). In the $0.2 \%$ time course study, hypertrophic GFAP-positive cells were readily detected in the corpus callosum within 1 week of cuprizone diet upon initiation of demyelination. Although the number of astrocytes during the first
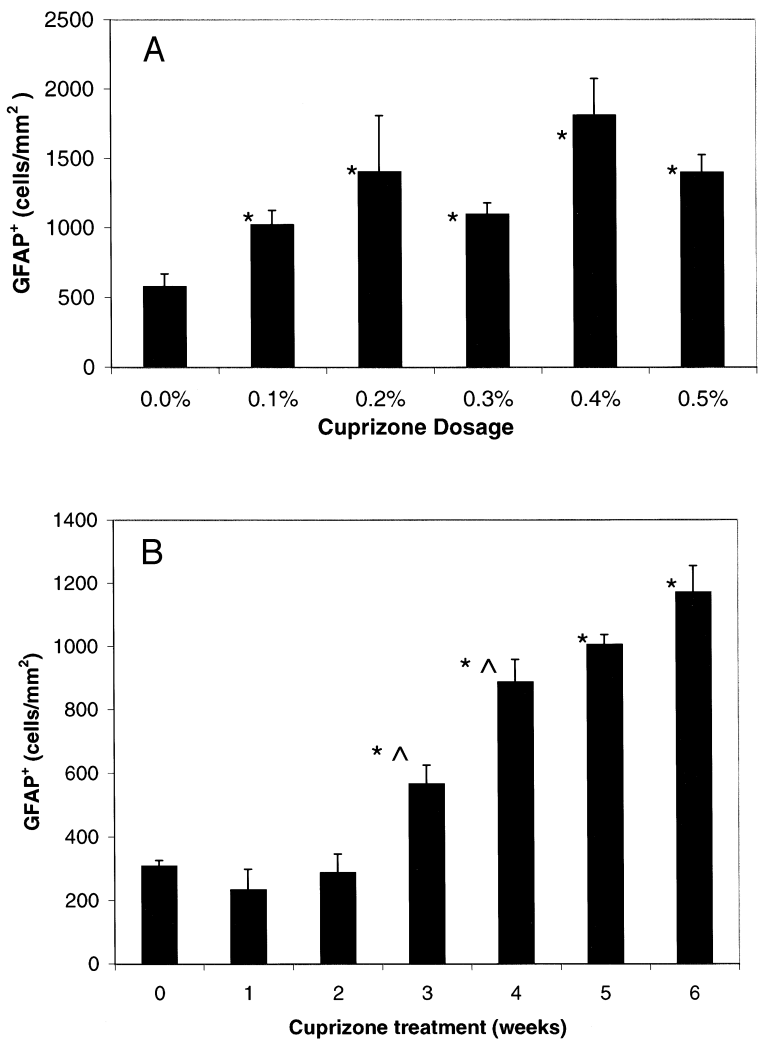

Fig. 7. Number of GFAP-staining astrocytes in the corpus callosum in C57BL/6 mice. Paraffin sections were cut at $5 \mu \mathrm{m}$ and stained with anti-GFAP antibody. Numbers of GFAP-staining cells containing nuclei were counted in the corpus callosum. (A) At 6 weeks with varying doses of cuprizone treatment, significantly increased numbers of astrocytes were apparent in mice treated with $0.2 \%$ cuprizone compared to untreated control animals $(* p<0.05)$. (B) During the $0.2 \%$ cuprizone time course, astrogliosis was first apparent at the 3rd week of cuprizone treatment, and it continued through the 6 th week of treatment $(* p<0.05)$. Astrogliosis initiated from the 2 nd to 3 rd week and progressed from 3rd to 4th week $(p<0.05)$ where it peaked and plateaued with the 6th week of cuprizone treatment. Each group is representative of an average of four mice, and the error bars represent standard deviation.

2 weeks of $0.2 \%$ cuprizone treatment did not appreciably increase, morphologic changes were apparent and presumably physiologic alterations were already undertaken. Thus, astrocytes contribute to the gliosis induced by cuprizone treatment and morphologic differences in astrocytes and their processes can be seen prior to demyelination and more interestingly, prior to gliosis.

\subsection{Appearance of microglia/macrophages upon induc- tion of demyelination}

Microglia/macrophages generally reside in the lesions of demyelination. Fig. 9A is the untreated control that showed the presence of RCA- $1^{+}$cells, markers of microglia/macrophage, in the corpus callosum is rare. In contrast, RCA-1 staining revealed a significant increase in the number of microglia/macrophages in the corpus callo- 
Fig. 8. Astrogliosis and morphological changes in $\mathrm{GFAP}^{+}$astrocytes upon initiation of demyelination. (A) Untreated control mice have little GFAP staining. (B) After 6 weeks of treatment with $0.2 \%$ cuprizone, there is an increased number of $\mathrm{GFAP}^{+}$astrocytes, indicated by arrows. Photomicrographs are shown at $600 \times$ magnification.

Fig. 9. Microglia/macrophages accumulate in the corpus callosum of C57BL/6 mice during demyelination. Paraffin sections were cut at $5 \mu \mathrm{m}$ and stained with RCA-1. (A) RCA-1 stain of the corpus callosum in the untreated mouse shows rare RCA-1 staining cells. (B) RCA-1 stain of the corpus callosum of a 6-week, $0.2 \%$ cuprizone-treated mouse indicates the extensive numbers of microglia/macrophages present in the corpus callosum. (C) As a confirmation of the RCA-1 stain, $30 \mu \mathrm{m}$ frozen coronal sections of the corpus callosum were stained for Mac-1. Mac-1 staining of coronal sections of untreated control mice indicates the presence of few microglia/macrophages with low immune reactivity in the normal brain. (D) Upon insult with cuprizone treatment, Mac-1 staining shows that microglia/macrophages with increased immune reactivity accumulate in large numbers in the corpus callosum, as shown here in the 6 -week, $0.2 \%$ cuprizone-treated mouse. Photomicrographs are shown at $600 \times$ magnification. 
sum as visualized in the brain section of a 6-week, $0.2 \%$ cuprizone-treated mouse (Fig. 9B). Similar results were observed with frozen sections immunostained with antibody to Mac-1, another marker for microglia/macrophages (Fig. 9C and D). Despite the use of $30 \mu \mathrm{m}$ sections that should permit the easier detection of rare microglia/macrophage cells, we observed very little staining in the untreated brain (Fig. 9C). However, microglia/macrophages were readily detected in the nearby cerebral cortex (data not shown). Examination of the frozen sections of the corpus callosum at 6-week, $0.2 \%$ cuprizone-treated C57BL/6 mice showed a large accumulation of $\mathrm{Mac}_{-} 1^{+}$microglia/macrophages located distinctly within the corpus callosum (Fig. 9D).

The quantification of microglia/macrophages and the appearance during the progression through demyelination had not been fully characterized previously. The presence of microglia/macrophages and the quantitative changes of microglia/macrophages in control vs. cuprizone-treated mice were visually evaluated with RCA-1 immunostained paraffin sections and Mac-1 immunostained frozen sections. In the dosage study, the number of microglia/macrophages in the corpus callosum (Fig. 10A) at all doses of cuprizone was significantly greater than the number of microglia/macrophages in the corpus callosum of untreated animals. Microglia/macrophages were present in the corpus callosum at the $0.1 \%$ dose (Fig. 10A) even when demyelination was not complete (Fig. 3). A substantially larger number of microglia/macrophages accumulated in the corpus callosum when greater than or equal to $0.2 \%$ cuprizone was present in the diet (Fig. 10A). Maximal microglial/macrophage accumulation into the corpus callosum appeared with doses of $0.4 \%-0.5 \%$ of cuprizone, doses when liver toxicity was observed; however, the number of microglia/macrophages at these higher doses were not significantly different from the $0.2 \%$ or $0.3 \%$ doses ( $p>0.05$, Student's $t$-test). In brains from untreated control animals, PAS or Mac-1-stained cells were not observed in the corpus callosum, cerebrum or cerebellum, although some ramified microglia/macrophages stained lightly with RCA-1. Thus, it appears that microglial/macrophage presence was prominent in regions of demyelination particularly at the $0.2 \%$ dose or greater.

In the chronological study of demyelination using the $0.2 \%$ dose of cuprizone, a significant increase in the number of microglia/macrophages was observed within the 1st week of cuprizone treatment (Fig. 10B). The early appearance of microglia/macrophages was prior to gross demyelination observed at week three (Fig. 5) and before significant astrogliosis (Fig. 7B). Furthermore, microglial/macrophage numbers increased significantly at week three $(p<0.05)$ and was correlated with the significant increase in progression of demyelination at 3 to 4 weeks (Fig. 5). In addition, dual staining with BrdU and RCA-1 in brain sections of cuprizone-treated mice revealed that the microglia/macrophages were proliferating
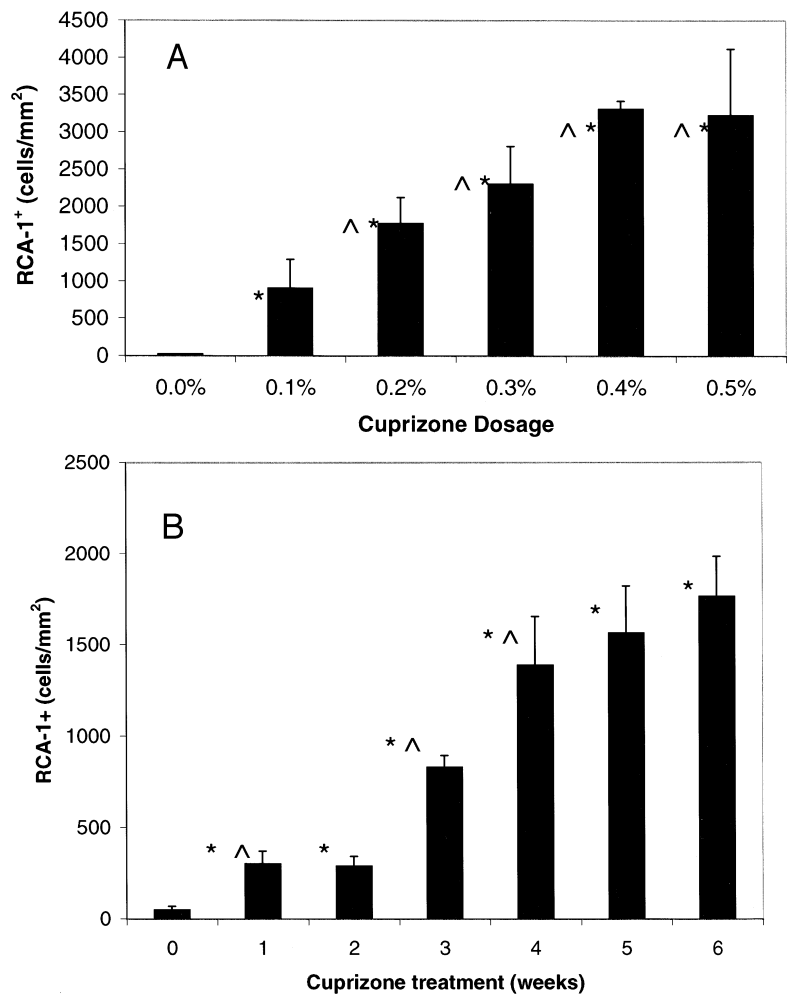

Fig. 10. Microglia/macrophage accumulation of the corpus callosum in C57BL/6 mice treated with cuprizone. (A) Animals were treated for 6 weeks with varying doses of cuprizone. Coronal brain sections of all mice treated with cuprizone were stained with RCA-1, an indicator of microglial presence. Numbers of microglia/macrophages in the corpus callosum were significantly higher $(* p<0.05)$ at all doses of cuprizone after 6 weeks of treatment when compared to the untreated control mouse. Numbers of microglia/macrophages in the corpus callosum of mice treated with $0.2 \%$ and greater were not significantly different from each other, although these higher doses showed significantly greater numbers of microglia/macrophages than the $0.1 \%$ dose $(p<0.05)$. (B) In the $0.2 \%$ cuprizone treatment time course study, microglia/macrophages appeared after the 1st week with initiation of cuprizone treatment. Significant microglial/macrophage accumulation compared to untreated mice was observed at weeks one through six $(* p<0.05)$. Additionally, a significant increase in microglial/macrophage accumulation compared to the previous week of treatment was noted at weeks one, three, and four ( $p<0.05$, Student's $t$-test). Each group in the graph is a mean of four mice, and the error bars represent standard deviation.

(data not shown). Because of the initial microglial/macrophage appearance at week one and week two prior to gross demyelination, and their continual increase in numbers in association with significant demyelination at week three through week six, microglia/macrophages may be actively participating in the demyelination process.

\section{Discussion}

We report here that the induction of demyelination by cuprizone treatment in C57BL/6 mice is associated with a microglial/macrophage presence. The dosage and time course of cuprizone treatment reported on $\mathrm{C} 57 \mathrm{BL} / 6$ mice 
are in drastic contrast to Swiss Webster mice. The advantage of establishing demyelination in the C57BL/6 mouse is that comparative analyses can be made with other mouse models for demyelination, such as the twitcher mouse, that are also on the same genetic (C57BL/6) background. Thus, similar hypotheses can be tested and corroborated in several models of demyelination, and that would lend credence to any discoveries and perhaps give insights to broad implications of any findings. Additionally, many genetically altered mice are on the $\mathrm{C} 57 \mathrm{BL} / 6$ genetic background; therefore, comparison of demyelination in these mutant mice to the wildtype C57BL/6 mouse may further implicate roles for additional effector molecules in demyelination without requiring backcrossing to strains susceptible to demyelination such as twitcher mice or SJL or $\mathrm{PL} / \mathrm{J}$ for $\mathrm{EAE}$.

The cuprizone-treated animals provide a model to study microglial/macrophage responses during the demyelinating process while the blood-brain barrier remains intact (Bakker and Ludwin, 1987; Kondo et al., 1987). In this study, we have three important findings. First, the $0.2 \%$ dose of cuprizone allowed demyelination to progress in the C57BL/6 mice without detrimental systemic toxic effects. Secondly, changes in morphology of $\mathrm{GFAP}^{+}$astroglia were concurrent with induction of demyelination, and astrogliosis was synchronous with demyelination and progressed until maximal demyelination. Finally and most importantly, microglia/macrophages appeared in the first 2 weeks upon induction with cuprizone and this was prior to gross demyelination observed at week three. Furthermore, microglia/macrophages increased in numbers until demyelination was complete. This last finding suggests that microglia/macrophages may not only be responding to demyelinated lesions, but they may also participate in the exacerbation of demyelination as a previous study using twitcher mice has suggested (Matsushima et al., 1994). Therefore, one can use cuprizone-treated C57BL/6 mice to study the specific demyelinating effects in the CNS, monitor the accumulation of the microglia/macrophages and corroborate findings with the twitcher mouse or other models for demyelination in order to attain broad insights about the progression of demyelination.

Previous studies on cuprizone treatment of mice used strains which were typically treated with $0.5 \%-0.6 \%$ dose of cuprizone (Suzuki and Kikkawa, 1969; Ludwin, 1978). In this report, when C57BL/6 mice were initially treated with $0.6 \%$ cuprizone diet, animals died within 2 to 3 weeks. C57BL/6 mice treated with slightly lower doses of cuprizone succumbed to the toxic affects of cuprizone as noted by lethargy, excessive weight loss, and/or liver toxicity (Figs. 1 and 2). The findings in this report indicate that a $0.2 \%$ dosage appears to affect oligodendrocytes and induce demyelination without extreme detrimental effects to the C57BL/6 mice. Demyelination was not extensive in C57BL $/ 6$ mice treated with the $0.1 \%$ dosage. Thus, it appears that $\mathrm{C} 57 \mathrm{BL} / 6$ mice possess a greater sensitivity to cuprizone treatment than the Swiss Webster mice, and all subsequent studies on demyelination with the cuprizone model in these mice should use the $0.2 \%$ cuprizone dosage.

Interestingly, morphological changes within astrocytes and accumulation of microglia/macrophages are synchronous with the initiation of demyelination. The number of astrocytes and microglia/macrophages continues to increase with the progression of demyelination. The increased numbers of microglia/macrophages and astrocytes plateaued once demyelination approaches completion around the 4 th to 5 th weeks of $0.2 \%$ cuprizone treatment. These data suggest that morphological changes occur in the brain immediately upon the onset of insult. Additionally, these data suggest that microglia/macrophages are not merely reacting to phagocytosed myelin debris, but may be directly participating as effector cells and causing damage to myelin structure and oligodendrocytes. This study provides a model in which microglia/macrophages may be effector cells that exacerbate the process of demyelination rather than cells which merely clear injured CNS tissue.

In vivo, MHC class II (Ia) molecules are found in the brain only on microglia/macrophages and serve as an indication of activation. We are currently addressing Ia expression in the cuprizone model. Our previous study suggests a model where Ia on microglia/macrophages serves to activate the cells which results in exacerbated clinical symptoms and neuropathology, and increased the number of microglia/macrophages (Matsushima et al., 1994). Thus, it would be interesting to test whether Ia on microglia/macrophages in the cuprizone model functions to exacerbate the neuropathology as it does in twitcher mice. These studies are currently underway utilizing the $I-A_{\beta}^{-/-}$mice, which are also on the C57BL/6 genetic background. We subsequently expect that this model for demyelination will be used in other genetic mouse mutants to address factors and/or molecules involved in demyelination, gliosis, or microglial/macrophage activation.

This cuprizone-induced demyelination provides testing of immunological impact on demyelination in an easily inducible, very predictable and consistent model. At the $0.2 \%$ cuprizone dose, evidence of demyelination on a microscopic scale is evident at 3 weeks of treatment and comes to completion at 6 weeks of treatment. Demyelination is localized and is most evident in the corpus callosum. Cuprizone induced demyelination is also reversible; therefore, this model makes it possible to study remyelination. These advantages of the cuprizone model, combined with the feasibility of analysis of demyelination in the C57BL/6 mouse will contribute important insights to the progression of demyelination as well as remyelination.

The findings in this study suggest that cuprizone treatment of C57BL/6 mice provide an ideal model to further test immune function during demyelinating disease. The time course and dosage used here provides a reasonable time period to assess net effects in the demyelinating 
process and should allow a number of genetically manipulated animals to be tested for specific function of molecules and cells in vivo. This model may provide an understanding of the functions of microglia/macrophages in demyelination and delineate mechanisms for microglia/macrophage interactions with the demyelinating process, but it will also allow the design of therapeutic protocols to be tested in vivo with relative ease.

\section{Acknowledgements}

We would like to thank Dr. R. Scott for the critical reading of this manuscript, and $\mathrm{K}$. Hedbacker for her excellent technical assistance. We would also like to acknowledge Peg Burchinal for her help with statistical analyses. GKM and this work was supported in part by the National Institute of Neurological Disorders and Stroke R29NS35372 and the National Multiple Sclerosis Society RG2754A1/2. J.P.-Y.T. was supported by a National Multiple Sclerosis Society grant RG-1785 and National Institute of Health grant NS34190. K.S. is supported in part by R01 NS24453 and P30-HD03110 (Mental Retardation Research Center Core).

\section{References}

Bakker, D.A., Ludwin, S.K., 1987. Blood-brain barrier permeability during cuprizone-induced demyelination: implication for the pathogenesis of immune-mediated demyelinating diseases. J. Neurol. Sci. $78,125-137$.

Blakemore, W.F., 1972. Observations on oligodendrocyte degeneration, the resolution of status spongiosus and remyelination in cuprizone intoxication in mice. J. Neurocytol. 1, 413-426.

Blakemore, W.F., 1973. Demyelination of the superior cerebellar peduncle in the mouse induced by cuprizone. J. Neurol. Sci. 20, 63-72.

Blakemore, W.F., 1974. Remyelination of the superior cerebellar peduncle in old mice following demyelination induced by cuprizone. J. Neurol. Sci. 22, 121-126.

Bruck, W., 1997. The role of macrophages in demyelination. Brain Pathol. 7, 741-752.

Bunge, M.B., Bunge, R.P., Ris, H., 1961. Ultrastructural study of remyelination in an experimental lesion in adult cat spinal cord. J. Biophys. Biochem. Cytol. 10, 67-94.

Cammer, W., Zhang, H., 1993. Atypical localization of the oligodendrocytic isoform (PI) of glutathione- $S$-transferase in astrocytes during cuprizone intoxication. J. Neurosci. Res. 36, 183-190.

Elsworth, S., Howell, J.M., 1973. Variation in the response of mice to cuprizone. Res. Vet. Sci. 14, 385-387.

Fujita, N., Ishiguro, H., Sato, S., Kurihara, T., Kuwano, R., Sakimura, K., Takahashi, Y., Miyatake, T., 1990. Induction of myelin-associated glycoprotein mRNA in experimental remyelination. Brain Res. 513, $152-155$.

Gledhill, R.F., Harrison, B.M., McDonald, W.I., 1973. Demyelination and remyelination following acute spinal cord compression. Exp. Neurol. 38, 472-487.

Harrison, B.M., McDonald, W.I., Ochoa, J., 1972. Remyelination in the central diphtheria toxin lesion. J. Neurol. Sci. 17, 293-302.
Hickey, W.G., Kimura, H., 1988. Perivascular microglial cells of the CNS are bone marrow-derived and present antigen in vivo. Science 239, 290-292.

Hirano, A., Levine, S., Zimmerman, H.M., 1968. Remyelination in the central nervous system after cyanide intoxication. J. Neuropathol. Exp. Neurol. 28, 234-245.

Komoly, S., Jeyasingham, M., Pratt, O., Lantos, P., 1987. Decrease in oligodendrocyte carbonic anhydrase activity preceding myelin degeneration in cuprizone induced demyelination. J. Neurol. Sci. 79, 141148.

Kondo, A., Nakano, T., Suzuki, K., 1987. Blood-brain barrier permeability in twitcher and cuprizone-intoxicated mice. Dev. Brain Res. 425, 186-190.

Lampert, P.W., 1965. Demyelination and remyelination in experimental allergic encephalomyelitis. J. Neuropathol. Exp. Neurol. 24, 371-385.

Lampert, P.W., 1967. Electron microscopic studies on ordinary and hyperacute experimental allergic encephalomyelitis. Acta Neuropathol. 9, 99-126.

Li, H., Newcombe, J., Groome, N., Cuzner, M.L., 1993. Characterization and distribution of phagocytic macrophages in multiple sclerosis plaques. Neuropathol. Appl. Neurobiol. 19, 214-223.

Ludwin, S.K., 1978. Central nervous system demyelination and remyelination in the mouse: an ultrastructural study of cuprizone toxicity. Lab. Invest. 39, 597-612.

Ludwin, S.K., 1981. Pathology of demyelination and remyelination. Adv. Neurol. 31, 123-168.

Martin, R., McFarland, H.F., McFarlin, D.E., 1992. Immunological aspects of demyelinating disease. Annu. Rev. Immunol. 10, 153-187.

Matsushima, G.K., Taniike, T., Glimcher, L.H., Grusby, M.J., Frelinger, J.A., Suzuki, K., Ting, J.P.-Y., 1994. Absence of MHC class II molecules reduces CNS demyelination, microglia/macrophage infiltration, and twitching in murine globoid cell leukodystrophy. Cell 78, 645-656.

Mattiace, L.A., Davies, P., Dickson, D.W., 1990. Detection of HLA-DR on microglia in post-mortem human brain is a function of clinical and technical factors. Am. J. Pathol. 136, 1101-1104.

McGeer, P.L., Itagaki, S., Boyes, B.E., McGeer, E.G., 1988a. Reactive microglia are positive for HLA-DR in the substantia nigra of Parkinson's and Alzheimer's disease brains. Neurology 38, 1285-1291.

McGeer, P.L., Itagaki, S., McGeer, E.G., 1988b. Expression of the histocompatibility glycoprotein HLA-DR in neurological disease. Acta Neuropathol. 76, 550-557.

Ohno, M., Higashi, Y., Suzuki, K., 1992. Microglial cell response to neuronal degeneration in the brain of brindled mouse. Dev. Brain Res. $67,37-45$.

Perlmutter, L.S., Scott, S.A., Barron, E., Chui, H.C., 1992. Ia-positive microglia in human brain: association with Alzheimer lesions. J. Neurosci. Res. 33, 549-558.

Prineas, J., Raine, C.S., Wisniewski, H., 1969. An ultrastructural study of experimental demyelination and remyelination: Part 3. Chronic experimental allergic encephalomyelitis in the central nervous system. Lab. Invest. $21,472-483$.

Raine, C.S., 1994. Multiple sclerosis: immune system molecule expression in the central nervous system. J. Neuropathol. Exp. Neurol. 53, 328-337.

Sidman, R.L., Angervine, J.B., Pierce, E.T., 1971. Atlas of the Mouse Brain and Spinal Cord. Commonwealth Fund Book. Harvard Univ. Press, Cambridge, MA, p. 38.

Smart, I., Leblond, C.P., 1961. Evidence for division and transformations of neuroglia cells in the mouse brain, as derived from radioautography after injection of thymidine- $\mathrm{H}^{3}$. J. Comp. Neurol. 116, 349-367.

Styren, S.D., Civin, W.H., Rogers, J., 1990. Molecular, cellular and pathological characterization of HLA-DR immunoreactivity in normal elderly and Alzheimer's disease brain. Exp. Neurol. 110, 93-104.

Suzuki, K., 1969. Giant hepatic mitochondria: production in mice fed with cuprizone. Science 163, 81-82.

Suzuki, K., Kikkawa, Y., 1969. Status spongiosus of CNS and hepatic 
changes induced by cuprizone (Bis-cyclohexanone oxalyldihydrazone). Am. J. Pathol. 54, 307-325.

Ting, J.P.-Y., Shigekawa, B., Linthicum, D.S., Weiner, L.P., Frelinger, J.A., 1981. Expression and synthesis of murine immune response associated (Ia) antigens by brain cells. Proc. Natl. Acad. Sci. USA 78, 3170-3174.

Tomimoto, H., Akiguchi, I., Akiyama, H., Kimura, J., Yanagihara, T., 1993. T-cell infiltration and expression of Ia antigen by macrophages and microglia in a heterogeneous group in leukoencephalopathy. Am. J. Pathol. 143, 579-586.

Traugott, U., Reinherz, E., Raine, C., 1983. Multiple sclerosis: distribution of T-cell subsets within active chronic lesions. Science 219, 308-310.
Walshe, J.M., 1995. Copper: not too little, not too much, but just right. J. R. Coll. Phys. London 29, 280-283.

Wisniewski, H., Raine, C.R., 1971. An ultrastructural study of experimental demyelination and remyelination: Part 5. Central and peripheral nervous system lesions caused by diphtheria toxin. Lab. Invest. $25,73-80$.

Yajima, K., Suzuki, K., 1979. Demyelination and remyelination in the rat CNS following ethidium bromide injection. Lab. Invest. 41, 385-392.

Zamvil, S.S., Steinman, L., 1990. The T-lymphocyte in experimental allergic encephalomyelitis. Annu. Rev. Immunol. 8, 579-621.

Zlotkin, S.H., Atkinson, S., Lockitch, G., 1995. Trace elements in nutrition for premature infants. Clin. Perinatol. 22, 223-240. 